\title{
The 2014 hypertension guidelines: implications for patients and practitioners in Asia
}

\author{
Fabio Angeli, ${ }_{1}^{1}$ Gianpaolo Reboldi, ${ }^{2}$ Paolo Verdecchia ${ }^{3}$
}

${ }^{1}$ Division of Cardiology and Cardiovascular

Pathophysiology, Hospital 'S.M. della Misericordia', Perugia, Italy

${ }^{2}$ Department of Medicine, University of Perugia, Perugia, Italy

${ }^{3}$ Department of Internal Medicine, Hospital of Assisi, Assisi, Italy

\section{Correspondence to}

Dr Paolo Verdecchia,

Department of Internal

Medicine, Hospital of Assisi,

Via Valentin Müller, 1, Assisi 06081, Italy; verdec@tin.it

Received 18 July 2015

Revised 1 September 2015

Accepted 3 September 2015

\section{CrossMark}

To cite: Angeli $F$,

Reboldi G, Verdecchia P.

Heart Asia 2015;7:21-25.

doi:10.1136/heartasia-2015-

010639

\section{ABSTRACT}

Hypertension is a global public health issue and a major cause of morbidity and mortality. Because of population growth and ageing, the number of people with uncontrolled hypertension rose from 600 million in 1980 to nearly 1 billion in 2008. Furthermore, the number of adults with hypertension in 2025 has also been predicted to increase by about $60 \%$ to a total of 1.56 billion. The prevalence of hypertension in most Asian countries has increased over the last 30 years and more dramatically in the last 10 years. Several factors contributed to such changes in Asia, but acculturation to Western lifestyle, modernisation and urbanisation are considered key contributing factors. There are some unique features in regards to cardiovascular risk in Asia. Specifically, Asian regions have disproportionately higher mortality and morbidity from stroke compared with Western countries. Furthermore, the relationship between blood pressure level and risk of stroke is stronger in Asia than in Western regions. Although evidence-based and qualified guidelines for hypertension diagnosis and management have been released recently from Europe and North America, the unique features of Asian patients with hypertension raise concerns in regards to the real clinical applicability of Western guidelines in Asian populations. Specifically, it is not yet clear to what extent the new blood pressure target proposed by Western guidelines for high risk and elderly hypertensive individuals apply to Asian populations.

\section{INTRODUCTION}

Hypertension is a global public health issue and a major cause of morbidity and mortality. ${ }^{1}$ Specifically, raised blood pressure (BP) is estimated to cause 7.5 million deaths, and it is reported to be responsible for almost 13\% of all deaths and 3.7\% of total disability-adjusted life years. ${ }^{1}$

Globally, the overall prevalence of raised BP in adults aged 25 years and over was around $40 \%$ in 2008. Because of population growth and ageing, the number of people with uncontrolled hypertension rose from 600 million in 1980 to nearly 1 billion in 2008. ${ }^{1}$ Furthermore, the number of adults with hypertension in 2025 has also been predicted to increase by about $60 \%$ to a total of 1.56 billion. $^{2}$

Across the WHO regions, the prevalence of hypertension is highest in Africa, where it is $46 \%$ for both sexes combined. The lowest prevalence of raised BP is in the WHO Region of the Americas at $35 \%$ for both sexes ${ }^{1}$ (figure 1, upper panel).

In the 10 countries of the Asian region from which data were available, the prevalence of high BP ranges from $19 \%$ in the Democratic People's Republic of Korea to $42 \%$ in Myanmar. ${ }^{1}$ Of note, the prevalence of hypertension is increasing in many countries of this region, and the much larger base Asian population results in a considerably larger absolute number of individuals affected ${ }^{3}$ (figure 1, upper panel).

When compared with Western countries, hypertension among Asian populations has unique features in terms of antihypertensive medication response, complications and outcomes. ${ }^{4}$

Although guidelines for the diagnosis and management of hypertension have been recently released from Europe ${ }^{5}$ and North America, ${ }^{6}$ Asian physicians need to consider nuances and features of their patients with hypertension ${ }^{3}$ before the translation of the recommendations from Western countries into clinical practice.

In this study, we briefly summarised the key elements from the most updated guidelines from Asia, and we critically reviewed and compared their similarities and differences with the recommendations from Europe ${ }^{5}$ and North America. ${ }^{6}$

\section{EPIDEMIOLOGY OF HYPERTENSION IN ASIA}

A variable prevalence of hypertension across Asian countries has been recently observed, with a number of countries with BP above the global average. $^{7}$

A recent systematic review and meta-analysis of 33 studies from member countries of the South Asian Association for Regional Cooperation $\left(\right.$ SAARC $^{7}$ showed that the overall prevalence of hypertension was around $27 \%$. The prevalence varied between the studies, ranging between $13.6 \%$ and $47.9 \%$, and was found to be higher in subjects from urban than rural areas. The prevalence of hypertension from the latest studies ${ }^{1} 7$ was: Bangladesh 17.9\%, Bhutan 23.9\%, India 31.4\%, Maldives 31.5\%, Mongolia 47\%, Nepal 33.8\%, Pakistan 25\%, Republic of Korea $30 \%$ and Sri Lanka $20.9 \%{ }^{\text {? }}$

The total number of patients with hypertension in Asia is expected to increase. For example, in China and India, the total number of patients with hypertension is expected to increase to more than 500 million in the next 10 years. ${ }^{4}$

These projections probably underestimate the real burden of hypertension to the Asian healthcare systems because the rising incidence of some hypertension-related risk factors (including obesity and diabetes mellitus) and unhealthy lifestyle in Asia have not been fully considered. ${ }^{4}$

\section{THE CASE OF STROKE}

Significant regional variations in the prevalence rates of coronary heart disease (CHD) and stroke have been documented in Asia. ${ }^{9-12}$ For example, the ascendancy of CHD over stroke has not been 
Figure 1 Reported prevalence of hypertension around the world (upper panel). The risk of stroke associated with higher systolic BP levels by age ( $<65$ vs $\geq 65$ years) and region (lower panel). Data from ref. 114. $\mathrm{BP}$, blood pressure. (Caucasian vs Asian) is also depicted

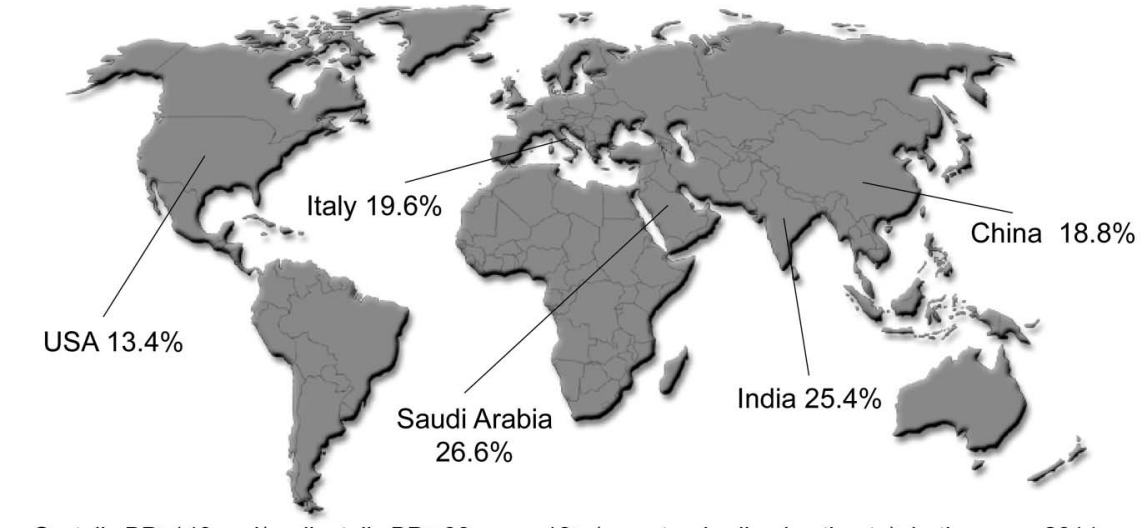

Systolic BP $\geq 140$ and/or diastolic BP $\geq 90$; ages 18+ (age standardized estimate); both sexes: 2014
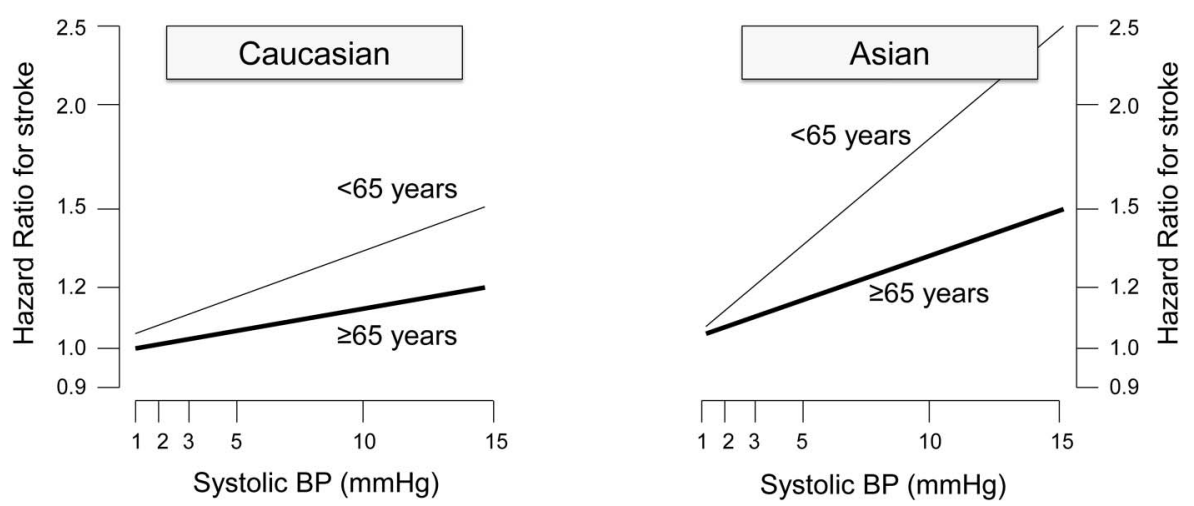

found in India. ${ }^{9}$ Although most Asian surveys have been limited to determining the actual prevalence and incidence rates of cardiovascular disease, the data accumulated so far are sufficient to declare that cardiovascular events are generally very much on rise. $^{13} 14$

Elevated BP is a key risk factor for cardiovascular disease in both Asian and Western populations. ${ }^{15} 16$ The Asia Pacific Cohort Studies Collaboration, which involved $>500000$ individuals followed for several years on average, has demonstrated a direct continuous association of usual levels of systolic and diastolic BP with the risks of CHD and stroke in both Caucasian and Asian populations. ${ }^{15}{ }^{16}$ However, the association of BP with stroke risk was about twice as steep as that with CHD risk, whereas the association with coronary risk was similar in the Asian and Caucasian populations; the association with stroke risk was generally steeper among Asians. ${ }^{10}{ }^{17}$ In other words, stroke is generally more common than CHD in most Asian populations, whereas the converse is seen in Western subjects, ${ }^{10}{ }^{17}$ and the linear relationship between systolic BP and stroke risk is markedly more pronounced in Asian than in Caucasian populations ${ }^{14}$ (figure 1, lower panel).

This appeared, in part, to be the consequence of the greater proportion of haemorrhagic strokes in the Asian population and the steeper association of $\mathrm{BP}$ with haemorrhagic rather than ischaemic stroke. There was also evidence of a steeper association of BP with haemorrhagic stroke among Asians compared with Caucasians. ${ }^{18}$

Quite recently, a meta-analysis and systematic overview was designed to evaluate randomised clinical trials carried out in Asia and to assess the effects of BP lowering on composite cardiovascular outcomes, as well as individual events, including stroke. ${ }^{19}$

It included 18 trials with 23215 and 21986 patients with hypertension in the intervention and reference groups, respectively. ${ }^{19}$ Of note, a subanalysis was conducted in a subgroup of eight trials that compared active treatment with placebo or intensive with less intensive BP control. BP (systolic/diastolic) decreased in the intervention group from 160/87 to 140/ $78 \mathrm{~mm} \mathrm{Hg}$ with a $-7 /-2 \mathrm{~mm} \mathrm{Hg}$ greater BP reduction than the reference group. ${ }^{19}$

Compared with the reference group, the intervention group had a lower risk of composite cardiovascular events (OR 0.73, $95 \%$ CI 0.66 to 0.81 ) and stroke (OR $0.71,95 \%$ CI 0.63 to 0.80). ${ }^{19}$

Notably, among all trials, a meta-regression analysis demonstrated that within-trial difference in systolic BP was inversely associated with composite cardiovascular events and stroke and that a $10 \mathrm{~mm} \mathrm{Hg}$ reduction in systolic BP was associated with a $30 \%$ reduced risk for stroke. ${ }^{19}$

\section{BP TARGET IN HIGH-RISK PATIENTS}

One of the most important differences between Asian and Western guidelines is the suggested target of BP levels for patients in whom the risk of stroke, vascular disease and renal failure is high. The last update of Western guidelines increased the target BP levels up to $140 / 90 \mathrm{~mm} \mathrm{Hg}$ for patients at highrisk of cardiovascular disease (with diabetes, cardiovascular or the previous recommendations to lower $\mathrm{BP}$ to $<130 / 80 \mathrm{~mm} \mathrm{Hg}$ in patients with diabetes are not supported by the extensive review of randomised controlled trials. Specifically, the Joint National Committee (JNC 8) and the European panels opted for a goal of $<140 / 90$ and $<140 / 85 \mathrm{~mm} \mathrm{Hg}$, respectively, in adult patients with hypertension and diabetes. ${ }^{5} 6$

However, the risk of cardiovascular disease in the Asian populations is significantly increased with uncontrolled BP and differs from that seen in patients from Western regions. ${ }^{20} 21$ Moreover, most Asian populations have a higher prevalence of renal disease). ${ }^{5}{ }^{6}$ Of note, these reappraisals ${ }^{5}{ }^{6}$ highlighted that 
stroke than of CHD and stroke is more closely associated with the level of BP treatment. ${ }^{14}$

These aspects are particularly relevant to Asian clinicians when diabetes, as a feature of increased cardiovascular risk, coexists with hypertension.

Hypertension and diabetes are highly prevalent among the Asian population, which may explain the high rate of stroke and coronary disease. ${ }^{18}$

It is a matter of fact that $\mathrm{BP}$ reduction improves outcome in adults with both hypertension and diabetes, but evidence supporting BP reduction to values close to or $<130 \mathrm{~mm} \mathrm{Hg}$ is scantier. $^{22}$

However, it should be emphasised that for those diabetic subjects at highest risk for stroke, lower BP may provide greater protection against stroke as shown in the Action to Control Cardiovascular Risk in Diabetes (ACCORD) BP trial. ${ }^{23}$

Specifically, the intensive lowering of the BP group (target systolic BP goal of $<120 \mathrm{~mm} \mathrm{Hg}$ ) showed a significant reduction of fatal and non-fatal stroke $(0.32 \%$ vs $0.53 \%$ in the intensive-therapy and standard-therapy groups, respectively). ${ }^{23}$ While this reduction in stroke did not contribute significantly to the primary composite outcome of the trial, it still has clinical relevance. $^{22} 23$

Considering these aspects, some Asian guidelines recommended more stringent BP targets in various groups of high-risk patients (including diabetics), although with some variations among these.

The 2015 Taiwan Society of Cardiology and Taiwan Hypertension Society guidelines $^{24}$ suggest a BP target of 130 / $80 \mathrm{~mm} \mathrm{Hg}$ for patients with diabetes, $\mathrm{CHD}$, proteinuric chronic kidney disease (CKD) and patients receiving antithrombotic therapy for stroke prevention.

The Japanese guidelines ${ }^{25}$ recommend a retention of the target of $<130 / 80 \mathrm{~mm} \mathrm{Hg}$ for patients with diabetes or CKD and microalbuminuria. Similarly, the Korean guidelines ${ }^{26}$ suggest tighter targets for high-risk patients, specifically $<140 /$ $85 \mathrm{~mm} \mathrm{Hg}$ for patients with diabetes and $<130 / 80 \mathrm{~mm} \mathrm{Hg}$ for patients with CKD and prominent albuminuria.

The Chinese guidelines from 2011 recommend a target of $<130 / 80 \mathrm{~mm} \mathrm{Hg}$ for patients with diabetes, CKD or CHD, but not for those with stroke. ${ }^{27}$

Guidelines from the Association of Physicians of India ${ }^{28}$ encourage the reduction of BP to around 140/80 and 130/ $85 \mathrm{~mm} \mathrm{Hg}$ in diabetics and in patients who have survived stroke, respectively.

\section{ELDERLY}

In many Asian countries, hypertension is very common among the elderly. For example, the prevalence in Chinese patients is $39 \%$ overall, $59.4 \%$ in patients aged $\geq 60$ years and $72.8 \%$ in those aged $\geq 75$ years. ${ }^{1} 29-31$

Furthermore, with a rapidly ageing population, the prevalence of hypertension and related cardiovascular morbidity in Asian patients continues to rise, placing a substantial and escalating social and economic burden on this region. In other words, more than two-thirds of the elderly population in Asia have to receive antihypertensive treatment in these countries. ${ }^{3} 32$

The last few years have seen a break in the previous consensus among Western guidelines in the treatment of the elderly. ${ }^{56}$ Guidelines from Europe ${ }^{5}$ and USA $^{6}$ broadly agree that a target of $<140 / 90 \mathrm{~mm} \mathrm{Hg}$ remains appropriate for uncomplicated patients with hypertension who are below the age of 60 years, but the American guidelines ${ }^{6}$ recommend raising this target to $<150 / 90 \mathrm{~mm} \mathrm{Hg}$ for patients over the age of 60 years. All these guidelines agree with the goal of $<150 / 90$ or $140-150$ / $90 \mathrm{~mm} \mathrm{Hg}$ for patients over the age of 80 years.

This threshold for the systolic reading is 10 points higher than in previous recommendations, and triggered controversy in the Asian medical community. ${ }^{19}$

As discussed above, the recent meta-analysis by Yano et al ${ }^{19}$ showed a benefit in terms of cardiovascular and stroke prevention when a BP target of $<140 / 80 \mathrm{~mm} \mathrm{Hg}$ is achieved in Asian patients with hypertension. Moreover, in the subgroup analysis of elderly patients, stroke risk was also decreased by intervention among trials comparing active treatment with placebo or intensive with less intensive BP control (OR 0.74, 95\% CI 0.58 to 0.93 ).

On the other hand, none of the available Asian intervention trials provided strong support for more ambitious BP targets in elderly patients with hypertension.

The Japanese trial to assess optimal systolic BP in elderly patients with hypertension (JATOS) ${ }^{33}$ was conducted in patients with hypertension aged 60-85 years and systolic BP $\geq 160 \mathrm{~mm} \mathrm{Hg}$. Patients were randomised to a more tight $(<140 \mathrm{~mm} \mathrm{Hg})$ or less tight $(140-160 \mathrm{~mm} \mathrm{Hg})$ systolic BP target and followed for 2 years. The primary end point, a composite of cardiovascular disease and renal failure, did not differ between the two groups. ${ }^{33}$

More recently, the picture has been complicated by the results of the Valsartan in Elderly Isolated Systolic Hypertension (VALISH) study. ${ }^{34}$ Overall, 3260 patients aged $70-84$ years were randomised to a more tight $(<140 \mathrm{~mm} \mathrm{Hg})$ or less tight $(140$ $150 \mathrm{~mm} \mathrm{Hg}$ ) systolic BP control. Valsartan was the first-line drug in all patients, with possible combination with other drugs, except other angiotensin receptor blockers, in order to achieve the assigned BP target. At the end of the study (median follow-up 3 years), systolic BP decreased by $5.4 \mathrm{~mm} \mathrm{Hg}$ more in the tight BP control group, but the primary outcome, a composite of fatal and non-fatal cardiovascular disease and renal failure, was only modestly reduced in the more tight control group (10.6 vs 12.0 per 1000 patient-years, $p=0.38$ ).

Given the uncertainty in the evidence for BP target in the elderly, the current guidance documents from the main Asian hypertension societies recommend a target of $<150 / 90 \mathrm{~mm} \mathrm{Hg}$ for older adults, but they differ in the ages at which this applies. $^{24-28}$

For the Chinese guidelines, ${ }^{27}$ this target applies for subjects over the age of 65 years; for the Japanese, ${ }^{25}$ it applies for 'latephase elderly'; the Korean guidelines ${ }^{26}$ recommend a target systolic BP between 140 and $150 \mathrm{~mm} \mathrm{Hg}$ in patients over the age of 65 years, while the Taiwan guidelines ${ }^{24}$ advocate a target BP of $<150 / 90 \mathrm{~mm} \mathrm{Hg}$ for elderly subjects over the age of 80 years.

\section{THE CHOICE OF BP-LOWERING DRUGS}

A number of clinical trials conducted in Asian patients have demonstrated that lowering BP is associated with reduced cardiovascular risk. ${ }^{19} 3536$

For example, the Systolic Hypertension in China (Syst-China) trial $^{35}$ showed that lowering BP with a step-wise active treatment with a calcium channel blocker (CCB) with or without an ACE inhibitor (ACE-I) and/or a thiazide diuretic significantly reduced the rate of cardiovascular events by $37 \%$, fatal and nonfatal strokes by $38 \%$, stroke mortality by $58 \%$ and total mortality by $39 \% .^{35}$

Similarly, the Felodipine Event Reduction (FEVER) trial $^{36}$ demonstrated that in patients with moderately complicated hypertension from China, even a difference in systolic/diastolic $\mathrm{BP}$ as small as $4 / 2 \mathrm{~mm} \mathrm{Hg}$, such as that induced by adding low-dose felodipine to low-dose hydrochlorothiazide, was 
associated with substantial reductions in the incidence of most types of cardiovascular events $(-33 \%$ for cardiovascular deaths and $-27 \%$ for fatal and non-fatal stroke). ${ }^{36}$

There is much agreement in the recommendations for drug treatment among the Asian guidelines. Those from China, Korea and Taiwan recommend five classes of first-line drugs (ACE-I, angiotensin receptor blockers (ARBs), beta-blockers (BBs), CCBs and diuretics); only the Japanese guidelines relegate BBs to second line.

Nevertheless, the most commonly used antihypertensive drugs in Asian countries are the CCBs, generally followed by ACE-Is and ARBs, although BBs are also frequently prescribed in Taiwan. ${ }^{37}$

Of note, a CCB-based regimen has been reported to achieve the highest rates of BP control, and it is recommended by the Asian Pacific Heart Association as the preferred strategy to improve BP control and to confront the aggravating epidemic of stroke. $^{38}$

In this context, some clinical studies in Asia and actively controlled trials and pooled analyses from Europe and North America showed a superior protection from stroke of CCBs when compared with other agents. ${ }^{38} 39$ Nevertheless, optimal treatment of hypertension often requires a combination therapy, and as highlighted by the Japanese Combination Therapy of Hypertension to Prevent Cardiovascular Events trial, ${ }^{40}$ it is very difficult to demonstrate that differences within BP-lowering drugs translate into differential effects with respect to hypertension-related cardiovascular complications.

\section{CONCLUSIONS}

The prevalence of hypertension in most Asian countries has dramatically increased over the last few years, and the optimal management of this condition is, therefore, an important task. ${ }^{1}$ Several factors contributed to such changes in Asia, but acculturation to Western lifestyle, modernisation and urbanisation are considered the key contributing factors. It is now well known that the social and environmental changes that accompany modernisation and urbanisation are important determinants of health. ${ }^{41-43}$ New health risk activities related to urbanisation and acculturation to Western lifestyle include saturated fat consumption, excessive alcohol intake, sedentary lifestyle and physical inactivity. ${ }^{41}$ These changes increase body weight, which is a wellestablished risk factor for the development of hypertension. ${ }^{41}$

Nevertheless, features of Asian hypertension remain different from those of Western countries. In particular, the relationship between BP level and stroke incidence is generally stronger in Asia, and there is a much higher incidence of haemorrhagic stroke in these populations (figure 1, lower panel).

The last few years have seen a break in the previous recommendation from Western guidelines to lower BP to $<130$ / $80 \mathrm{~mm} \mathrm{Hg}$ in high-risk patients. However, features of Asian hypertension raise doubts concerning the real clinical applicability of Western guidelines in Asian populations. In view of the evidence that Asian populations have substantial differences from Western countries in the characteristics of hypertension and related cardiovascular disease burden, we agree with current recommendations from Asian societies to lower BP to $<130 / 80 \mathrm{~mm} \mathrm{Hg}$ for patients with hypertension who are at increased risk of stroke.

Regarding treatment of hypertension in the elderly, it should be noted that the linear relationship between systolic BP and stroke risk is steeper among Asian than Caucasian populations and that such a relationship appears to be unaffected by age (figure 1, lower panel). This may be explained by the greater proportion of strokes that were haemorrhagic in the Asian population and by the closer association observed between BP and haemorrhagic rather than ischaemic stroke.

Furthermore, the high prevalence of hypertension among the elderly justifies a specific approach towards the treatment of hypertension in this group of patients in Asia.

Taken together, these findings support the concerns of experts that raising the threshold for systolic BP to $150 \mathrm{~mm} \mathrm{Hg}$ could lead to an increased incidence of stroke in Asia, and it makes sense to adopt a target BP of $<140 / 90 \mathrm{~mm} \mathrm{Hg}$ in the elderly provided it can be achieved without undue treatment burden.

The paucity of data on the correct definition of the most appropriate BP target in the elderly, highlighted by the few available trials, should be perceived as a stimulus for future research in Asia, not as an argument for questioning the benefit of treatment. $^{44}$

Finally, although antihypertensive drug treatment has demonstrated efficacy in lowering BP and reducing cardiovascular events, hypertension remains an area of significant unmet medical needs in Asia. Control of BP is often suboptimal in clinical practice with rates among Asian patients with hypertension rarely exceeding $50 \% ;{ }^{37}$ furthermore, it is not entirely clear whether reduction in cardiovascular events may be achieved irrespective of the class of antihypertensive agent used.

Thus, selecting appropriate BP-lowering drugs is a particular challenge in Asian populations because of the lack of sufficient Asia-specific evidence.

Contributors All authors contributed to the interpretation of data, drafting of the manuscript, critical revision of the manuscript for important intellectual content and final approval.

Competing interests None declared.

Provenance and peer review Commissioned; internally peer reviewed.

\section{REFERENCES}

1 WHO Global Health Observatory. Prevalence of raised blood pressure: situations and trends. http://wwwwhoint/gho/ncd/risk_factors/blood_pressure_prevalence_text/en/ (accessed 10 Jul 2015).

2 Kearney PM, Whelton M, Reynolds K, et al. Global burden of hypertension: analysis of worldwide data. Lancet 2005;365:217-23.

3 Chung N, Baek S, Chen MF, et al. Expert recommendations on the challenges of hypertension in Asia. Int J Clin Pract 2008;62:1306-12.

4 Kearney PM, Whelton M, Reynolds K, et al. Worldwide prevalence of hypertension: a systematic review. J Hypertens 2004;22:11-19.

5 Mancia G, Fagard R, Narkiewicz K, et al. 2013 ESH/ESC guidelines for the management of arterial hypertension: the Task Force for the Management of Arterial Hypertension of the European Society of Hypertension (ESH) and of the European Society of Cardiology (ESC). Eur Heart J 2013;34:2159-219.

6 James PA, Oparil S, Carter BL, et al. 2014 evidence-based guideline for the management of high blood pressure in adults: report from the panel members appointed to the Eighth Joint National Committee (JNC 8). JAMA 2014;311:507-20.

7 Neupane D, McLachlan CS, Sharma R, et al. Prevalence of hypertension in member countries of South Asian Association for Regional Cooperation (SAARC): systematic review and meta-analysis. Medicine (Baltimore) 2014;93:e74.

8 Singh RB, Suh IL, Singh VP, et al. Hypertension and stroke in Asia: prevalence, control and strategies in developing countries for prevention. J Hum Hypertens 2000;14:749-63.

9 Nag T, Ghosh A. Cardiovascular disease risk factors in Asian Indian population: a systematic review. J Cardiovasc Dis Res 2013;4:222-8.

10 Ueshima $H$. Explanation for the Japanese paradox: prevention of increase in coronary heart disease and reduction in stroke. J Atheroscler Thromb 2007;14:278-86.

11 Wang JG, Li Y. Characteristics of hypertension in the Chinese population. Curr Hypertens Rep 2012;14:410-15.

12 Wong MC, Tam WW, Cheung CS, et al. Drug adherence and the incidence of coronary heart disease- and stroke-specific mortality among 218,047 patients newly prescribed an antihypertensive medication: a five-year cohort study. Int I Cardiol 2013;168:928-33. 
13 Das SK, Banerjee TK. Stroke: Indian scenario. Circulation 2008;118:2719-24.

14 Perkovic V, Huxley R, Wu Y, et al. The burden of blood pressure-related disease: a neglected priority for global health. Hypertension 2007;50:991-7.

15 Lawes CM, Rodgers A, Bennett DA, et al. Asia Pacific Cohort Studies Collaboration. Blood pressure and cardiovascular disease in the Asia Pacific region. I Hypertens 2003;21:707-16.

16 Woodward M, Barzi F, Martiniuk A, et al. Asia Pacific Cohort Studies Collaboration. Cohort profile: the Asia Pacific Cohort Studies Collaboration. Int J Epidemiol 2006;35:1412-16.

17 Ishikawa Y, Ishikawa J, Ishikawa S, et al. Jichi Medical School Cohort Investigators Group. Prehypertension and the risk for cardiovascular disease in the Japanese general population: the Jichi Medical School Cohort Study. J Hypertens 2010;28:1630-7.

18 Woodward M, Huxley H, Lam TH, et al. Asia Pacific Cohort Studies Collaboration. A comparison of the associations between risk factors and cardiovascular disease in Asia and Australasia. Eur J Cardiovasc Prev Rehabil 2005;12:484-91.

19 Yano Y, Briasoulis A, Bakris GL, et al. Effects of antihypertensive treatment in Asian populations: a meta-analysis of prospective randomized controlled studies (CARdiovascular protectioN group in Asia: (ARNA). J Am Soc Hypertens 2014;8:103-16.

20 Arima H, Murakami Y, Lam TH, et al. Asia Pacific Cohort Studies Collaboration. Effects of prehypertension and hypertension subtype on cardiovascular disease in the Asia-Pacific Region. Hypertension 2012;59:1118-23.

21 Shimamoto K, Fujita T, Ito $\mathrm{S}$, et al. J-HEALTH Study Committees. Impact of blood pressure control on cardiovascular events in 26,512 Japanese hypertensive patients: the Japan Hypertension Evaluation with Angiotensin II Antagonist Losartan Therapy ( J-HEALTH) study, a prospective nationwide observational study. Hypertens Res 2008;31:469-78.

22 Angeli F, Verdecchia P, Reboldi G. Intensive blood pressure control in obese diabetic patients: clinical relevance of stroke prevention in the ACCORD trial. Expert Rev Cardiovasc Ther 2012;10:1467-70.

23 Group AS, Cushman WC, Evans GW, et al. Effects of intensive blood-pressure control in type 2 diabetes mellitus. N Engl J Med 2010;362:1575-85.

24 Chiang CE, Wang TD, Ueng KC, et al. 2015 guidelines of the Taiwan Society of Cardiology and the Taiwan Hypertension Society for the management of hypertension. J Chin Med Assoc 2015;78:1-47.

25 Shimamoto K, Ando K, Fujita T, et al. Japanese Society of Hypertension Committee for Guidelines for the Management of Hypertension. The Japanese Society of Hypertension Guidelines for the Management of Hypertension (JSH 2014) Hypertens Res 2014;37:253-390.

26 Shin DS, Park JB, Kim KB, et al, Guideline Committee of the Korean Society of Hypertension. 2013 Korean Society of Hypertension guidelines for the management of hypertension. Part 1—epidemiology and diagnosis of hypertension. Clin Hypertens 2015;21:1.

27 Liu LS, Writing Group of Chinese Guidelines for the Management of Hypertension. [2010 Chinese guidelines for the management of hypertension]. Zhonghua Xin Xue Guan Bing Za Zhi 2011;39:579-615.
28 Association of Physicians of India. Indian guidelines on hypertension (I.G.H.) - III. 2013. J Assoc Physicians India 2013;61:6-36.

29 Sheng CS, Liu M, Kang YY, et al. Prevalence, awareness, treatment and control of hypertension in elderly Chinese. Hypertens Res 2013;36:824-8.

30 Gu D, Reynolds K, Wu X, et al. InterASIA Collaborative Group. The International Collaborative Study of Cardiovascular Disease in ASIA. Prevalence, awareness, treatment, and control of hypertension in china. Hypertension 2002;40:920-7.

31 Malhotra R, Chan A, Malhotra C, et al. Prevalence, awareness, treatment and control of hypertension in the elderly population of Singapore. Hypertens Res 2010;33:1223-31.

32 Lee JH, Yang DH, Park HS, et al. Investigators HYpertension-Diabetes Daegu Initiative Study Investigators. Incidence of hypertension in Korea: 5-year follow-up study. J Korean Med Sci 2011;26:1286-92.

33 Group JS. Principal results of the Japanese trial to assess optimal systolic blood pressure in elderly hypertensive patients (JATOS). Hypertens Res 2008;31:2115-27.

34 Ogihara T, Saruta T, Rakugi H, et al. Valsartan in Elderly Isolated Systolic Hypertension Study Group. Target blood pressure for treatment of isolated systolic hypertension in the elderly: valsartan in elderly isolated systolic hypertension study. Hypertension 2010;56:196-202.

35 Liu L, Wang JG, Gong L, et al. Comparison of active treatment and placebo in older Chinese patients with isolated systolic hypertension. Systolic Hypertension in China (Syst-China) Collaborative Group. J Hypertens 1998;16:1823-9.

36 Liu L, Zhang Y, Liu G, et al. FEVER Study Group. The Felodipine Event Reduction (FEVER) Study: a randomized long-term placebo-controlled trial in Chinese hypertensive patients. J Hypertens 2005;23:2157-72.

37 Park JB, Kario K, Wang JG. Systolic hypertension: an increasing clinical challenge in Asia. Hypertens Res 2015;38:227-36.

38 Wang JG, Kario K, Lau T, et al. Asian Pacific Heart Association. Use of dihydropyridine calcium channel blockers in the management of hypertension in Eastern Asians: a scientific statement from the Asian Pacific Heart Association. Hypertens Res 2011;34:423-30.

39 Verdecchia P, Reboldi G, Angeli F, et al. Angiotensin-converting enzyme inhibitors and calcium channel blockers for coronary heart disease and stroke prevention. Hypertension 2005;46:386-92.

40 Matsuzaki M, Ogihara T, Umemoto S, et al. Combination Therapy of Hypertension to Prevent Cardiovascular Events Trial G. Prevention of cardiovascular events with calcium channel blocker-based combination therapies in patients with hypertension: a randomized controlled trial. J Hypertens 2011;29:1649-59.

41 Angeli F, Reboldi G, Verdecchia P. Hypertension around the world: new insights from developing countries. J Hypertens 2013;31:1358-61.

42 Angeli F, Reboldi G, Verdecchia P. "From apennines to andes:" does body mass index affect the relationship between age and blood pressure? Hypertension 2012;60:6-7.

43 Angeli F, Reboldi G, Verdecchia P. Modernization and hypertension: is the link changing? Hypertens Res 2013;36:676-8.

44 Angeli F, Reboldi G, Verdecchia P. "The lower the BP the better" paradigm in the elderly: vanished by VALISH? Hypertension 2010;56:182-4. 